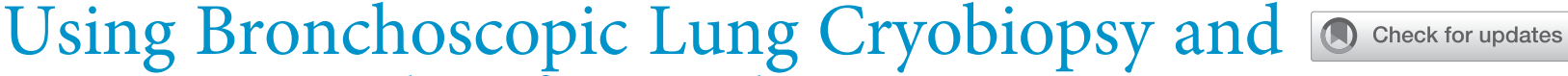 a Genomic Classifier in the Multidisciplinary Diagnosis of Diffuse Interstitial Lung Diseases
}

Fayez Kheir, MD; Ala Alkhatib, MD; Gerald J. Berry, MD; Philip Daroca, MD; Lisa Diethelm, MD; Reinaldo Rampolla, MD; Shigeki Saito, MD; David L. Smith, MD; David Weill, MD; Marjorie Bateman, MD; Ramsy Abdelghani, MD;

and Joseph A. Lasky, MD

BACKGROUND: Challenges remain for establishing a specific diagnosis in cases of interstitial lung disease (ILD). Bronchoscopic lung cryobiopsy (BLC) has impacted the diagnostic impression and confidence of multidisciplinary discussions (MDDs) in the evaluation of ILD. Reports indicate that a genomic classifier (GC) can distinguish usual interstitial pneumonia (UIP) from non-UIP.

RESEARCH QUESTION: What is the impact of sequentially presented data from BLC and GC on the diagnostic confidence of MDDs in diagnosing ILD?

STUDY DESIGN AND METHODS: Two MDD teams met to discuss 24 patients with ILD without a definitive UIP pattern. MDD1 sequentially reviewed clinical-radiologic findings, BLC, and GC. MDD2 sequentially reviewed GC before BLC. At each step in the process the MDD diagnosis and confidence level were recorded.

RESULTS: MDD1 had a significant increase in diagnostic confidence, from $43 \%$ to $93 \%$ ( $P=$ .023), in patients with probable UIP after the addition of GC to BLC. MDD2 had an increase in diagnostic confidence, from $27 \%$ to $73 \%(P=.074)$, after the addition of BLC to GC. The concordance coefficients and percentage agreement of categorical idiopathic pulmonary fibrosis (IPF) and non-IPF diagnoses were as follows: GC vs MDD1: 0.92, 96\%; GC vs MDD2: 0.83 , 92\%; BLC1 vs MDD1: $0.67,83 \%$; BLC2 vs MDD2: $0.66,83 \%$.

INTERPRETATION: GC increased diagnostic confidence when added to BLC for patients with a probable UIP pattern, and in appropriate clinical settings can be used without BLC. In contrast, BLC had the greatest impact regarding a specific diagnosis when the likelihood of UIP was considered low following clinical-radiographic review.

CHEST 2020; 158(5):2015-2025

KEY WORDS: bronchoscopy; cryobiopsy; genomic classifier; interstitial lung diseases; multidisciplinary discussion

FOR EDITORIAL COMMENT, SEE PAGE 1814

ABBREVIATIONS: $\mathrm{BLC}=$ bronchoscopic lung cryobiopsy; $\mathrm{CHP}=$ chronic hypersensitivity pneumonitis; DLCO = diffusing capacity of the lungs for carbon monoxide; GC = genomic classifier; HRCT = highresolution CT; ILD = interstitial lung disease; IPF = idiopathic pulmonary fibrosis; $\mathrm{MDD}=$ multidisciplinary discussion; $\mathrm{OP}=$ organizing pneumonia; SLB = surgical lung biopsy; UIP = usual interstitial pneumonia

AfFiliations: From the Division of Pulmonary and Critical Care, Department of Medicine (Drs Kheir, Alkhatib, Saito, Bateman,
Abdelghani, and Lasky), Tulane University School of Medicine, New Orleans, LA; the Department of Pathology (Dr Berry), Stanford University School of Medicine, Stanford, CA; the Department of Pathology (Dr Daroca), Tulane University School of Medicine, New Orleans, LA; the Department of Radiology (Dr Diethelm), Ochsner Clinic Foundation, New Orleans, LA; the Department of Pulmonology (Dr Rampolla), Ochsner Multi-Organ Transplant Institute, New Orleans, LA; the Department of Radiology (Dr Smith), Louisiana State 


\section{Take-home Point}

We found that a genomic classifier that distinguishes usual interstitial pneumonia (UIP) from non-UIP in tissue obtained by transbronchial biopsy had a meaningful impact on the diagnostic confidence in the multidisciplinary diagnosis of UIP regardless of bronchoscopic lung cryobiopsy.

Bronchoscopic lung cryobiopsy (BLC) has become an accepted modality in many centers to obtain lung tissue samples from patients with diffuse interstitial lung disease (ILD). In other centers surgical lung biopsy (SLB) remains the routine diagnostic approach. ${ }^{1-3} \mathrm{~A}$ report of the European IPF registry indicated that cryobiopsy had been used in $92 \%$ of patients as compared with $8 \%$ for SLB among all patients who were referred for a lung biopsy. ${ }^{4}$ BLC is a relatively safe procedure with significantly lower morbidity and mortality compared with SLB, and delivers a diagnostic yield of about $80 \%$ in ILD. ${ }^{1-3}$ A study showed that BLC has a meaningful impact on the diagnostic confidence in the multidisciplinary diagnosis of idiopathic pulmonary fibrosis (IPF). ${ }^{2}$

A newly introduced genomic classifier (GC) applied to lung tissue obtained by transbronchial biopsy can distinguish usual interstitial pneumonia (UIP) from non-UIP and can aid physicians and multidisciplinary teams in confirming the diagnosis of IPF in patients without a definite UIP pattern on high-resolution CT (HRCT) imaging. ${ }^{5}$ Current guidelines recommend multidisciplinary discussion (MDD) to reach a consensus diagnosis following clinical-radiologic evaluation as well as after the addition of histopathologic information for ILD. ${ }^{6,7}$ However, whether there is an advantage in using both BLC and GC in the context of an MDD remains unanswered. The main aim of this study was to address the impact of sequentially presenting data from BLC and GC on the diagnostic confidence of MDDs in diagnosing ILD.

\section{Methods}

\section{Patient Selection}

This study was approved by the Tulane University Institutional Review Board (IRB No. 2018-1749) with a waiver of informed consent as this was a retrospective examination of clinically indicated data. Informed consent for BLC and GC was obtained from patients per institutional policy.

Consecutive patients, 40 years and older, with clinical and radiologic features of ILD, but without a definite UIP pattern on HRCT imaging per referring physicians and our experienced pulmonologists, as defined by patterns described by Raghu et $\mathrm{al}^{7}$ pertaining to clinical practice guidelines, were enrolled into this study. The indication for BLC was based on a decision made by the ILD and interventional pulmonologists who evaluated the patients at our center.

Patients with acute exacerbation of ILD, uncorrectable coagulopathy, or who were receiving antiplatelet therapy (except for aspirin) or anticoagulation that could not be withheld; had a platelet count less than $50,000 / \mu \mathrm{L}$, an $\mathrm{FVC}<35 \%$, and/or diffusing capacity of the lungs for carbon monoxide (DLCO) less than $35 \%$ of their predicted

University, New Orleans, LA; and the Weill Consulting Group (Dr Weill), New Orleans, LA.

Drs Kheir and Alkhatib contributed equally to this manuscript.

Part of this article has been presented at the CHEST Annual Meeting 2019, October 19-23, 2019, New Orleans, LA.

FUNDING/SUPPORT: The authors have reported to CHEST that no funding was received for this study.

CORRESPONDENCE TO: Joseph A. Lasky, MD, 1430 Tulane Ave, Box 8509, New Orleans, LA 70112; e-mail: jlasky@tulane.edu

Copyright (c) 2020 American College of Chest Physicians. Published by Elsevier Inc. All rights reserved.

DOI: https://doi.org/10.1016/j.chest.2020.05.532

values; had an estimated systolic pulmonary artery pressure greater than $50 \mathrm{~mm} \mathrm{Hg}$ on transthoracic echocardiography; had hypoxemia requiring nasal oxygen $>4 \mathrm{~L} / \mathrm{min}$ at rest; who were receiving mechanical ventilation; or who were pregnant were excluded from the study.

\section{Bronchoscopic Lung Cryobiopsy}

All transbronchial lung cryobiopsies were performed with a flexible bronchoscope by an experienced interventional pulmonologist in the bronchoscopy suite, with the patient under general anesthesia. Patients were intubated with a size 8.5 - to $9.0-\mathrm{mm}$ endotracheal tube. The biopsy site was chosen by the interventional bronchoscopist, taking into consideration the HRCT scan appearance of each patient. All cryobiopsies were performed under fluoroscopic guidance with radial endobronchial ultrasound (UM-S20-17S; Olympus America Inc.), using a 1.9-mm-diameter cryoprobe (ERBOKRYO; Erbe USA Inc.) as previously described. ${ }^{8}$ A Fogarty balloon occlusion catheter 5.0 (Fogarty; Edwards Lifesciences) was positioned at the entrance of the targeted segmental bronchus and used prophylactically after each cryobiopsy. ${ }^{8}$ All patients underwent BAL for WBC differential.

\section{Pathologic Assessment}

Processing of the cryobiopsy specimen was performed in a fashion similar to that described by Hetzel et al. ${ }^{1}$ BLC was considered nondiagnostic when the histopathologic criteria were insufficient to define a characteristic histopathologic pattern. Before the MDD meeting, the pathologists (P. D. and G. J. B.) examined the pathologic specimens independently and recorded their individual impressions in a blinded manner.

\section{Genomic Classifier Sample Processing}

Five transbronchial lung biopsy samples (typically two from the upper lobe and three from the lower lobe) were collected under fluoroscopic guidance for molecular testing from all participants, using radial jaw pulmonary biopsy forceps. The samples were taken from the same 


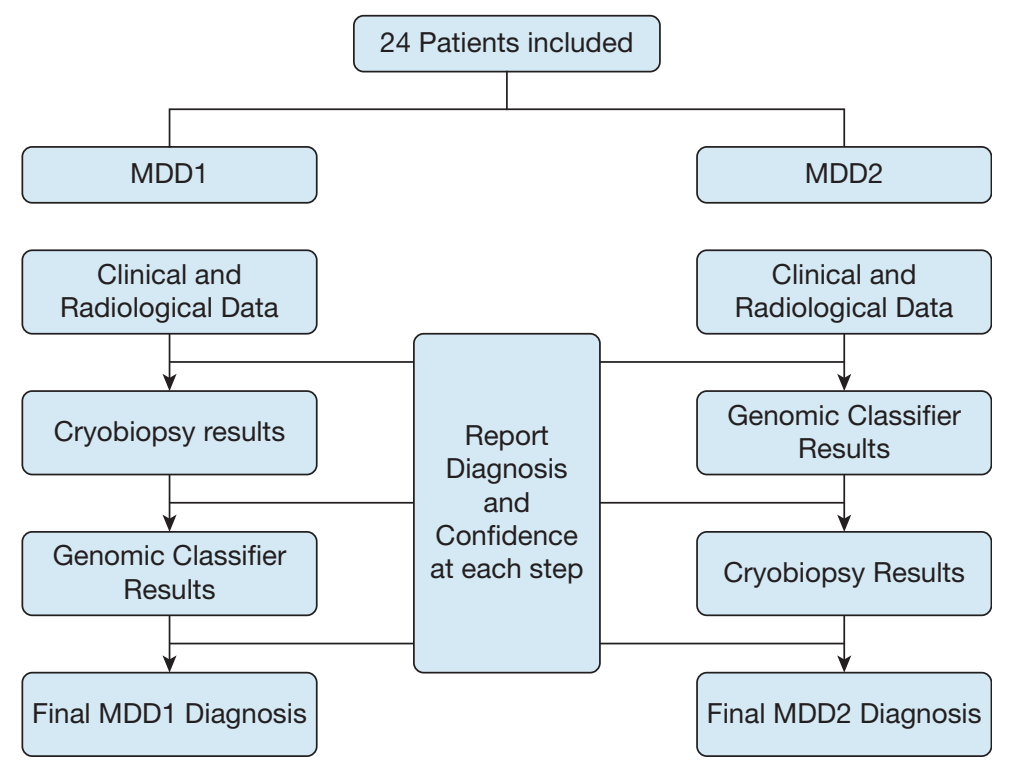

Figure 1 - Organizational scheme. $M D D=$ multidisciplinary discussion.

lung lobes sampled for the BLC. The transbronchial lung biopsy samples were provided to Veracyte via an early-access program (Envisia Genomic Classifier; Veracyte) and were processed for RNA sequencing and analysis, using their standardized laboratory procedure, to identify a UIP pattern. ${ }^{9}$

\section{Data Collection}

A standard form was used to collect clinical information including symptoms, environmental exposures, comorbid illnesses, medication use, smoking history, family history, physical examination findings, serologic data, and procedure-associated complications. Pulmonary function data (spirometry, lung volumes, and DLCO) and HRCT scans taken within 6 months of BLC and GC were reviewed.

\section{Organizational Scheme}

All MDD participants were educated about BLC and GC, including review of a recent relevant article, ${ }^{5}$ before discussing the cases. The methodology described by Flaherty et $\mathrm{al}^{6}$ was used to estimate the diagnostic impact of BLC and GC on the diagnosis of ILD in the MDD process. The ILD cases were reviewed by two separate independent MDDs that comprised two ILD experts, one chest radiologist, and one pulmonary pathologist. MDD1 sequentially reviewed clinical-radiologic findings, then biopsy results along with the lavage WBC differential, and finally the GC result. MDD2 sequentially reviewed the clinical-radiologic findings, then the GC result before the BLC pathology report, along with the lavage cell differential. MDD1 differed from MDD2 in two aspects. First, a physician in MDD1 interviewed and examined each patient. Second, MDD1 was composed of physicians who have previously published articles involving RNA-Seq (J. A. L.) and BLC (J. A. L.). The organizational scheme is summarized in Figure 1.

The study was designed to evaluate whether the sequential addition of BLC and/or GC information influenced the diagnostic confidence of the MDD. Participants met at the Tulane Lung Center in New Orleans and were given information anonymously in a stepwise fashion by two presenters (A. A. and R. A.) as outlined in Figure 1. At each step, participants recorded their diagnostic impression before and after group discussion. Participants could change their diagnostic impression and/or confidence level at each step of the review process. Each MDD participant expressed their confidence level of an ILD diagnosis, using the ontology of Ryerson et $\mathrm{al},{ }^{10}$ as follows: "unclassifiable ILD" (0\%-50\% diagnostic likelihood), provisional diagnosis of IPF with low confidence (51\%$69 \%$ diagnostic likelihood), provisional diagnosis of IPF with high confidence (70\%-89\% diagnostic likelihood), and definite diagnosis of IPF (90\%-100\% diagnostic likelihood). We defined high confidence of diagnosis of fibrotic ILD as $\geq 70 \%$. After MDDs were held for all cases, the groups recorded a consensus diagnosis and confidence level. Consensus diagnosis was achieved when all participants in the MDD agreed on the ILD diagnosis.

\section{Complications}

Bleeding related to cryobiopsy was categorized as mild (requiring bronchoscopic suctioning), moderate (requiring intrabronchial instillation of epinephrine or ice-cold saline), or severe (requiring termination of the procedure, blood transfusion, or prolonged intubation requiring monitoring in the ICU). ${ }^{1}$ Chest radiography was performed on all patients within 3 hours of the procedure to evaluate for procedure-related pneumothorax as well as other complications.

\section{Statistical Analyses}

Prism 8 (GraphPad Software) was used for statistical analysis. Descriptive statistics were used to summarize clinical and radiologic characteristics. Cohen's $\kappa$ coefficient and percentage agreement with their 95\% CIs were computed for GC vs BLC1 and BLC2; GC vs final diagnoses in both multidisciplinary discussions; two radiologists; two histopathologists; and final diagnoses reported in both MDDs. The McNemar test was used to compare the proportions of confidence level changes during the MDD process. A $\kappa$ value $\leq 0.20$ indicates slight agreement; 0.21 to 0.40 , fair agreement; 0.41 to 0.60 , moderate agreement; 0.61 to 0.80 , substantial agreement; and $>0.80$, excellent agreement. A $P$ value $<$ .05 was defined as significant. 


\section{Results}

Twenty-four patients who underwent BLC and transbronchial biopsy for GC testing between June 2018 and February 2019 at Tulane University Medical Center were included in the study. Clinical characteristics and baseline demographics are shown in Table 1 . The majority of patients were men (54.2\%) and white (83.3\%), with a mean age of 65.7 years. The most common presenting symptoms were shortness of breath (87.5\%) and cough (83.3\%). Pulmonary function testing revealed restrictive lung disease with a mean FVC of $64.6 \%$ and decreased Dico (49.1\%). The median number of cryobiopsies per procedure was three (two to six), and the mean area of the samples was $40.2 \pm 16.3 \mathrm{~mm}^{2}$. Four patients (16.7\%) had mild bleeding, three patients (12.5\%) had moderate bleeding, and no patient had severe bleeding. Two patients (8\%) developed a pneumothorax and required small-bore chest tube thoracostomy and hospitalization for 2 days.

The proportion of high-confidence diagnoses before providing the histopathology or GC results was similar between MDD1 and MDD2 (13\% vs 17\%, respectively). The overall agreement between the two chest radiologists (D. L. S. and L. D.) in each MDD was 79\% (95\% CI, 57\%-92\%) ( $\kappa, 0.64 ; 95 \%$ CI, 0.36-0.92). The histopathology slides were reviewed by two expert lung pathologists (P. D. and G. J. B.); the histopathology assessment was nondiagnostic in 10 of 24 of the cases (42\%) reviewed by the first pathologist and in eight of 24 of the cases $(33 \%)$ reviewed by the second pathologist (Table 2). Blinded assessment for the same samples by the two pathologists indicated an overall agreement of 83\% (95\% CI, 62\%-95\%) ( $\kappa, 0.77$; 95\% CI, 0.56-0.98). Adding BLC to the clinical-radiologic diagnosis in MDD1 increased the proportion of high-confidence diagnoses from $13 \%$ to $46 \%(P=.013)$, whereas adding the GC to the clinical-radiologic diagnosis in MDD2 increased the proportion of high-confidence diagnoses from $17 \%$ to $29 \%(P=.24)$. The overall agreement between the final MDD1 diagnosis and the final MDD2 diagnosis was $73 \%$ (95\% CI, 62\%-95\%) ( $\kappa, 0.77$; 95\% CI, 0.58-0.96). However, the overall agreement regarding the categorical diagnosis of IPF vs non-IPF was $100 \%$. The GC was positive in all patients with a final diagnosis of IPF whereas histopathology assessment was either UIP or favoring UIP in $69 \%$ of the cases in both MDDs.

\section{Outcomes}

The radiologist assessment indicated that 14 patients had a probable UIP pattern and 10 patients had an indeterminate pattern in MDD1 as compared with three definitive, 11 probable, and 10 indeterminate for UIP in MDD2.

For MDD1, 12 of 14 patients with probable UIP had a positive GC result and received a final diagnosis of IPF, and the other two patients with negative GC results received diagnoses of chronic hypersensitivity pneumonitis (CHP) and autoimmune ILD (Table 2). The clinical-radiologic diagnosis initially was unclassifiable in seven cases. The number of unclassifiable cases decreased to four with BLC and to three after adding the GC results. For MDD2, 13 of 14 patients with a definitive or probable UIP pattern had a positive GC result: 11 received a final diagnosis of IPF whereas the other two patients received diagnoses of $\mathrm{CHP}$ and organizing pneumonia (OP) (Table 2). The clinical-radiologic diagnosis initially was unclassifiable in nine cases. The number of unclassifiable cases decreased to eight with the GC results and to five after adding the BLC results.

The proportion of high-confidence diagnoses was significantly higher in MDD1 after adding the GC results to the BLC results for all enrolled patients ( $46 \%$ vs $75 \% ; P=.023$ ). The increase in confident diagnoses was higher among those with probable UIP ( $43 \%$ to $93 \% ; P=.023$ ) and among patients receiving a final diagnosis of IPF (31\% vs $92 \%$; $P=.013$ ) (Fig 2); however, the diagnostic confidence remained the same (60\%) among patients with CT scans that were indeterminate for UIP after adding the GC results to the $\mathrm{BLC}$ results in MDD1. Adding BLC or GC changed the clinical-radiologic diagnosis in four of 24 and two of 24 cases, respectively.

The proportion of high-confidence diagnoses for all patients minimally increased in MDD2 after providing the GC results alone ( $17 \%$ vs $29 \% ; P=.24$ ) but was significantly higher after providing the histopathology results $(29 \%$ vs $67 \% ; P=.007)$. Among patients with probable UIP, the proportion of high-confidence diagnoses following provision of the GC and histopathology results was $0 \%$ vs $27 \%(P=.25)$ and $27 \%$ vs $73 \%(P=.074)$, respectively. A similar pattern was noticed among patients with a final diagnosis of IPF (Fig 2). In patients with CT scans that were indeterminate for UIP, the proportion of highconfidence diagnoses remained the same, at $10 \%$, after providing the GC results without histopathology to the MDD2; however, it increased ( $10 \%$ to $50 \%$; $P=.134$ ) after providing MDD2 with the histopathology 
TABLE 1$]$ Demographic and Clinical Characteristics of Patients Undergoing Cryobiopsy

\begin{tabular}{|c|c|}
\hline Demographic/Clinical Variable & $\begin{array}{c}\text { Patients } \\
\text { Undergoing } \\
\text { Cryobiopsy }\end{array}$ \\
\hline Age, mean (SD), y & $65.7 \pm 10.7$ \\
\hline Men, No. (\%) & $13(54.2)$ \\
\hline White, No. (\%) & $20(83.3)$ \\
\hline \multicolumn{2}{|l|}{ Time course of symptoms, No. (\%) } \\
\hline$>1 y$ & $16(66.7)$ \\
\hline$<1 \mathrm{y}$ & $4(16.7)$ \\
\hline Unknown & $4(16.7)$ \\
\hline \multicolumn{2}{|l|}{ Presenting symptoms, No. (\%) } \\
\hline Shortness of breath & $21(87.5)$ \\
\hline Cough & $20(83.3)$ \\
\hline Xerostomia & $7(29.2)$ \\
\hline Weight loss & $3(12.5)$ \\
\hline Raynaud phenomenon & $3(12.5)$ \\
\hline Chest pain & $2(8.3)$ \\
\hline Fevers and chills & $2(8.3)$ \\
\hline Night sweats & $2(8.3)$ \\
\hline \multicolumn{2}{|l|}{ Medical comorbidities, No. (\%) } \\
\hline Asthma & $2(8.3)$ \\
\hline COPD & $7(29.2)$ \\
\hline OSA & $7(29.2)$ \\
\hline Gastroesophageal reflux disease & $14(58.3)$ \\
\hline Coronary artery disease & $4(16.7)$ \\
\hline Hypertension & $16(66.7)$ \\
\hline Diabetes mellitus type 2 & $6(25)$ \\
\hline Cancer & $2(8.3)$ \\
\hline Chronic kidney disease & $2(8.3)$ \\
\hline Congestive heart failure & $2(8.3)$ \\
\hline Depression or anxiety & $4(16.7)$ \\
\hline Current or former smoker & $18(75)$ \\
\hline \multicolumn{2}{|l|}{ Family history, No. (\%) } \\
\hline Autoimmune disease & $4(16.7)$ \\
\hline Pulmonary disease (not fibrosis) & $2(8.3)$ \\
\hline Pulmonary fibrosis & $5(20.8)$ \\
\hline \multicolumn{2}{|l|}{$\begin{array}{l}\text { Environmental and occupational } \\
\quad \text { exposures, No. (\%) }\end{array}$} \\
\hline Mold & $7(29.2)$ \\
\hline Birds & $5(20.8)$ \\
\hline Other pets & $7(29.2)$ \\
\hline Asbestos & $3(12.5)$ \\
\hline Hay, animal feed & $3(12.5)$ \\
\hline Clothing production materials & $2(8.3)$ \\
\hline Construction & $4(16.7)$ \\
\hline Medications known to cause ILD, No. (\%) & $4(16.7)$ \\
\hline
\end{tabular}

(Continued)
TABLE 1 ] (Continued)

\begin{tabular}{|l|c}
\hline Demographic/Clinical Variable & \multicolumn{1}{|c}{$\begin{array}{c}\text { Patients } \\
\text { Undergoing } \\
\text { Cryobiopsy }\end{array}$} \\
\hline Abnormal serology workup, No. (\%) & $10(41.6)$ \\
\hline BMI, mean (SD) & $33.3 \pm 9.7$ \\
\hline $\begin{array}{l}\text { Supplemental oxygen requirement, yes, } \\
\quad \text { No. (\%) }\end{array}$ & $5(20.8)$ \\
\hline TLC \% predicted, mean (SD) & $72.4 \pm 16.3$ \\
\hline FEV 1 /FVC, mean (SD) & $79.6 \pm 5.2$ \\
\hline FVC \% predicted, mean (SD) & $64.6 \pm 14.8$ \\
\hline FEV $1 \%$ predicted, mean (SD) & $67.2 \pm 13.4$ \\
\hline DLCo \% predicted, mean (SD) & $49.1 \pm 13.4$ \\
\hline \hline
\end{tabular}

DLco = diffusing capacity of the lungs for carbon monoxide; ILD = interstitial lung disease; TLC $=$ total lung capacity.

interpretation. Adding BLC or GC changed the clinicalradiologic diagnosis in seven of 24 and one of 24 cases, respectively.

In the categorical diagnosis of UIP vs non-UIP for MDD1, the concordance between GC and the assessment of histologic samples by the expert lung pathologist (P. D.) was 79\% (95\% CI, 57\%-92\%) $(\kappa, 0.60$; 95\% CI, 0.29-0.91). The discordance between the GC and histopathology assessment occurred in five cases, all with positive GC results. Four of them had nondiagnostic histopathology. The final diagnosis was IPF in all four patients with nondiagnostic histopathology. CHP, based on exposure history, pops and squeaks on examination, and air trapping on HRCT scanning, was the MDD1 diagnosis for the fifth patient, who also had diagnostic CHP histopathology (Table 2).

In the categorical diagnosis of UIP vs non-UIP for MDD2, the concordance between GC and the assessment of histologic samples by the expert lung pathologist (G. J. B.) was 75\% (95\% CI, 53\%-89\%) ( 0.53 ; 95\% CI, 0.20-0.85). The discordance with the pathologist occurred in six cases (all with positive GC results), the pathology assessment in three of the cases was nondiagnostic histopathology, and the other three cases were reported as aspiration, OP, and CHP. The final diagnosis in these patients was IPF in four and the other two received diagnoses of OP and CHP (Table 2).

There was an overall agreement of $88 \%$ (95\% CI, 67\%$97 \%)(\kappa, 0.75 ; 95 \%$ CI, 0.48-1.00) between the GC result and the reported MDD1 diagnosis assigned before providing the GC results to the group. The overall agreement between the GC result and the final MDD1 diagnosis of UIP or non-UIP was $96 \%$ (95\% CI, 
TABLE 2 ] Genomic Classifier, High-Resolution CT Imaging, Bronchoscopic Lung Cryobiopsy, and MDD for the Two Groups

\begin{tabular}{|c|c|c|c|c|c|c|c|c|c|}
\hline Case & $\begin{array}{c}\mathrm{GC} \\
\text { Diagnosis }\end{array}$ & $\begin{array}{l}\text { HRCT Scan } \\
\text { Diagnosis } \\
\text { (MDD1) }\end{array}$ & $\begin{array}{l}\text { Initial } \\
\text { Confidence }^{a} \\
\text { (MDD1) }\end{array}$ & $\begin{array}{l}\text { BLC Diagnosis } \\
\text { (MDD1) }\end{array}$ & $\begin{array}{c}\text { Final } \\
\text { Diagnosis } \\
\text { (MDD1) }\end{array}$ & $\begin{array}{l}\text { HRCT Scan } \\
\text { Diagnosis } \\
\text { (MDD2) }\end{array}$ & $\begin{array}{l}\text { Initial } \\
\text { Confidence }^{a} \\
\text { (MDD2) }^{\text {(MDD }}\end{array}$ & $\begin{array}{c}\text { BLC } \\
\text { Diagnosis } \\
\text { (MDD2) }\end{array}$ & $\begin{array}{l}\text { Final } \\
\text { Diagnosis } \\
\text { (MDD2) }\end{array}$ \\
\hline 1 & Positive & Probable UIP & Provisional low & UIP & IPF & Probable UIP & Provisional low & UIP & IPF \\
\hline 2 & Negative & Indeterminate & Unclassifiable ILD & $\begin{array}{l}\text { Organizing } \\
\text { pneumonia }\end{array}$ & $\begin{array}{l}\text { Organizing } \\
\text { pneumonia }\end{array}$ & Indeterminate & $\begin{array}{l}\text { Unclassifiable } \\
\text { ILD }\end{array}$ & $\begin{array}{l}\text { Organizing } \\
\text { pneumonia }\end{array}$ & $\begin{array}{l}\text { Organizing } \\
\text { pneumonia }\end{array}$ \\
\hline 3 & Negative & Indeterminate & Unclassifiable ILD & Unclassifiable & Unclassifiable & Indeterminate & $\begin{array}{l}\text { Unclassifiable } \\
\text { ILD }\end{array}$ & Unclassifiable & Unclassifiable \\
\hline 4 & Positive & Probable UIP & Provisional low & UIP & IPF & Probable UIP & Provisional low & UIP & IPF \\
\hline 5 & Negative & Probable UIP & Provisional low & Autoimmune ILD & $\begin{array}{l}\text { Autoimmune } \\
\text { ILD }\end{array}$ & Probable UIP & Provisional low & $\mathrm{CHP}$ & $\mathrm{CHP}$ \\
\hline 6 & Negative & Indeterminate & $\begin{array}{l}\text { Provisional high } \\
\text { (autoimmune) }\end{array}$ & Unclassifiable & $\begin{array}{l}\text { Autoimmune } \\
\text { ILD }\end{array}$ & Indeterminate & $\begin{array}{l}\text { Unclassifiable } \\
\text { ILD }\end{array}$ & RB-ILD & RB-ILD \\
\hline 7 & Positive & Probable UIP & Provisional low & UIP & IPF & Probable UIP & Provisional low & UIP & IPF \\
\hline 8 & Negative & Probable UIP & Provisional low & Unclassifiable & $\mathrm{CHP}$ & Indeterminate & $\begin{array}{l}\text { Unclassifiable } \\
\text { ILD }\end{array}$ & Unclassifiable & Unclassifiable \\
\hline 9 & Positive & Probable UIP & Provisional low & UIP & IPF & Probable UIP & Provisional low & UIP & IPF \\
\hline 10 & Positive & Probable UIP & Provisional low & UIP & IPF & UIP & High & UIP & IPF \\
\hline 11 & Positive & Probable UIP & Provisional low & UIP & IPF & Probable UIP & Provisional low & UIP & IPF \\
\hline 12 & Negative & Indeterminate & Unclassifiable ILD & Sarcoidosis & Sarcoidosis & Indeterminate & $\begin{array}{l}\text { Unclassifiable } \\
\text { ILD }\end{array}$ & Sarcoidosis & Sarcoidosis \\
\hline 13 & Positive & Probable UIP & Provisional low & UIP & IPF & Probable UIP & Provisional low & $\begin{array}{l}\text { Organizing } \\
\text { pneumonia }\end{array}$ & $\begin{array}{l}\text { Organizing } \\
\text { pneumonia }\end{array}$ \\
\hline 14 & Positive & Indeterminate & Unclassifiable ILD & Unclassifiable & CPFE & Indeterminate & $\begin{array}{l}\text { Unclassifiable } \\
\text { ILD }\end{array}$ & Unclassifiable & CPFE \\
\hline 15 & Positive & Probable UIP & Provisional low & UIP & IPF & Probable UIP & Provisional low & UIP & IPF \\
\hline 16 & Positive & Probable UIP & Provisional low & Unclassifiable & IPF & Probable UIP & Provisional low & Aspiration & IPF \\
\hline 17 & Positive & Indeterminate & $\begin{array}{l}\text { Provisional high } \\
\text { (CHP) }\end{array}$ & $\begin{array}{l}\text { Hypersensitivity } \\
\text { pneumonitis }\end{array}$ & $\mathrm{CHP}$ & Probable UIP & Provisional low & $\mathrm{CHP}$ & $\mathrm{CHP}$ \\
\hline 18 & Positive & Probable UIP & Provisional low & UIP & IPF & Probable UIP & Provisional low & UIP & IPF \\
\hline 19 & Negative & Indeterminate & Unclassifiable ILD & NSIP & NSIP & Indeterminate & $\begin{array}{l}\text { Provisional } \\
\text { high }\end{array}$ & NSIP & NSIP \\
\hline 20 & Positive & Probable UIP & Provisional low & Unclassifiable & IPF & UIP & High & Unclassifiable & IPF \\
\hline
\end{tabular}

(Continued) 


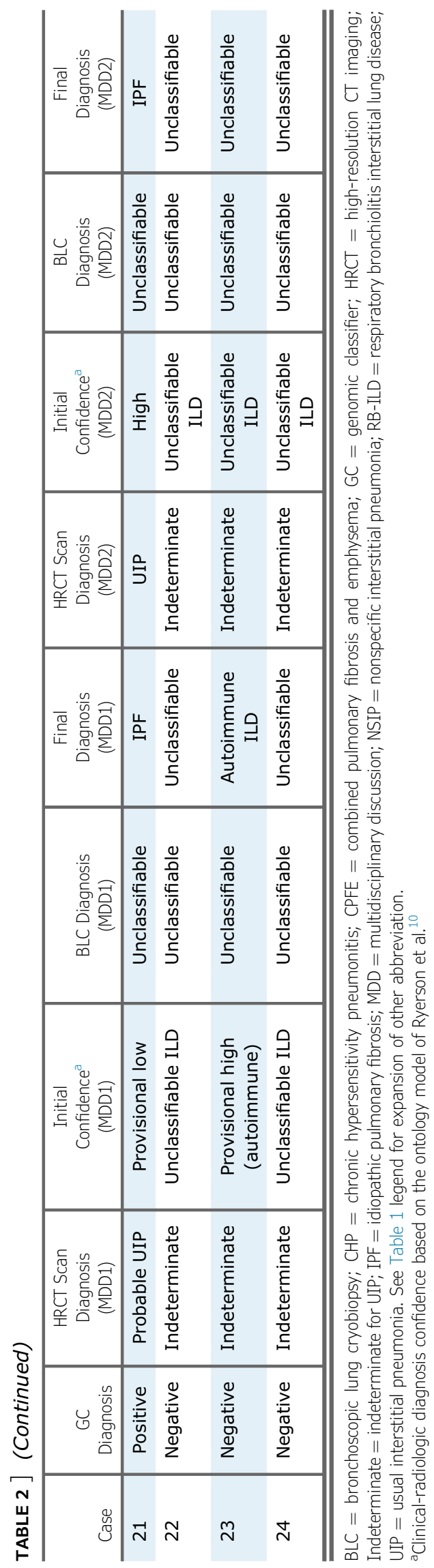

77\%-100\%) ( $\kappa, 0.92 ; 95 \% \mathrm{CI}, 0.75-1.00)$, as compared with an overall agreement of $83 \%$ (95\% CI, $62 \%-95 \%)$ $(\kappa, 0.67 ; 95 \% \mathrm{CI}, 0.38-0.97)$ between the histopathology and final MDD1 diagnosis of UIP (Fig 3). In patients with a radiologic pattern of probable UIP, the overall agreement of UIP or non-UIP between GC and the final MDD1 diagnosis was $100 \%$ vs $79 \%$ between histopathology and the final MDD1 diagnosis (Fig 4). The GC was positive in all 13 patients receiving a final diagnosis of IPF; however, the pathologist made a highconfidence diagnosis of UIP in only three of 13 cases. A UIP diagnosis with low confidence was made in seven cases and an unclassifiable diagnosis was made in three cases. The clinical-radiologic diagnosis initially was unclassifiable in seven cases. The number of unclassifiable cases decreased to four with transbronchial lung cryobiopsy and to three after adding the GC results.

The overall agreement between the GC and the final MDD2 diagnosis of UIP or non-UIP was $92 \%$ (95\% CI, $72 \%-99 \%)(\kappa, 0.83$; 95\% CI, 0.62-1.00) as compared with an overall agreement of $83 \%$ (95\% CI, 62\%-95\%) ( $\kappa$, 0.66; 95\% CI, 0.36-0.96) between the histopathology and final MDD2 classification (Fig 3). In patients with a radiologic pattern of definitive UIP or probable UIP, the overall agreement of IPF or non-IPF between GC and the final MDD2 diagnosis was $86 \%$ vs $79 \%$ between histopathology and the final MDD2 diagnosis (Fig 4). The GC was positive in all 12 patients receiving a final diagnosis of IPF; however, the pathologist made a high confidence diagnosis of UIP in only five of 12 cases. A UIP diagnosis with low confidence was made in four cases and an unclassifiable diagnosis was made in three cases.

\section{Discussion}

Achieving a diagnosis of ILD is challenging even in the setting of an MDD. The current clinical practice guidelines for IPF, published in 2018 as a joint statement from the American Thoracic Society, European Respiratory Society, Japanese Respiratory Society, and the Latin American Thoracic Society, recommend a multidisciplinary approach that involves physicians, radiologists, and pathologists. ${ }^{7}$ Recently, a GC that uses RNA sequencing data from transbronchial lung biopsy samples identified a video-assisted thoracic surgery biopsy UIP pattern with a specificity and sensitivity of $88 \%$ and $70 \%$, respectively, and was recently approved by the Food and Drug Administration to help physicians and multidisciplinary teams in confirming the diagnosis 

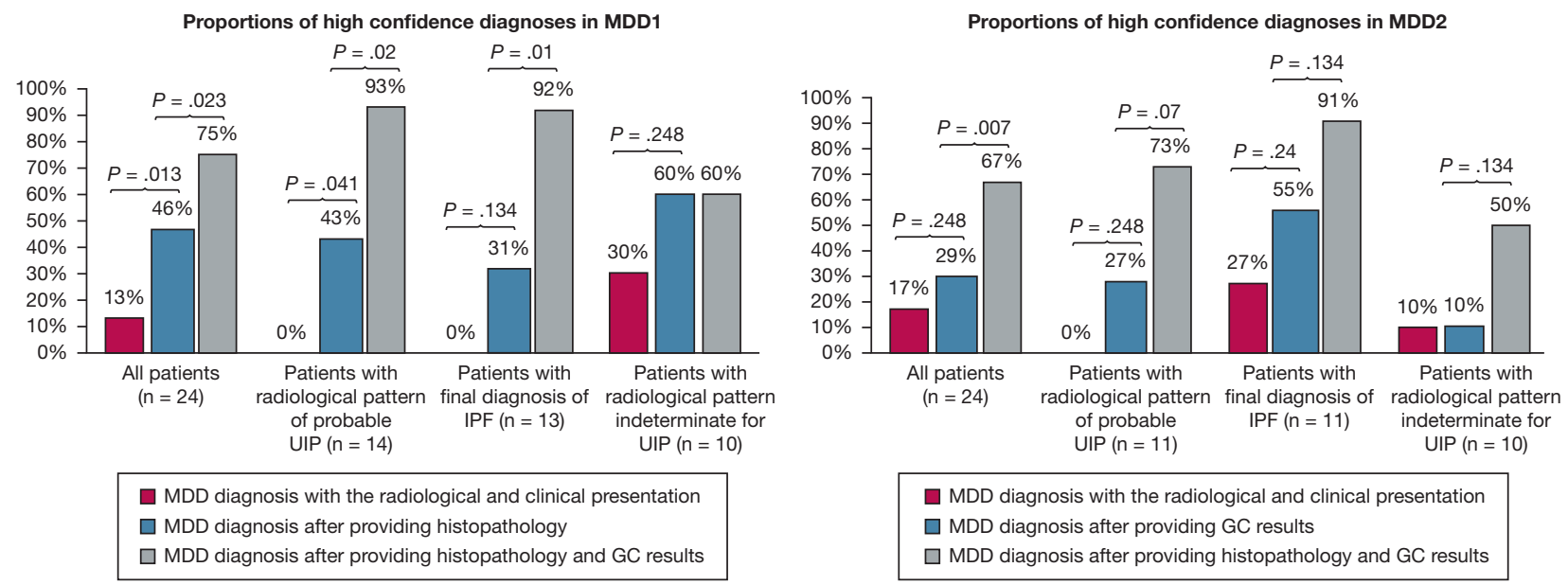

Figure 2 - Comparison of diagnostic confidence for MDD1 (left) and MDD2 (right). IPF = idiopathic pulmonary fibrosis; UIP = usual interstitial pneumonia. See Figure 1 legend for expansion of other abbreviation.

of UIP in patients without a definitive radiologic pattern for UIP. ${ }^{5}$

In the appropriate clinical setting, biopsy may not be needed for patients with a radiologic pattern of probable UIP if the clinical context suggests IPF. ${ }^{711}$ However, some patients with a radiologic pattern of probable UIP may have abnormal, but nonspecific, autoantibodies, a significant environmental or work exposure history, or medication regimens that are associated with ILD (Table 1), and thus may need additional testing and/or MDD work to establish whether they have IPF. In this study, we tested the impact of BLC and GC on the multidisciplinary process in cases in which the HRCT scan indicated probable UIP with confounding clinical factors, or was indeterminate for UIP. We found that the confidence levels of MDD1 increased considerably when the GC was added to the BLC, as the proportion of IPF cases diagnosed with a high degree of confidence increased from $31 \%$ to $92 \%$. In contrast, there was a moderate increase (51\% to $91 \%$ ) when the BLC results were added to the GC.

Adding BLC alone to the clinical-radiologic diagnosis in MDD1 had more impact on the proportion of highconfidence diagnoses ( $13 \%$ to $46 \%$ ) than adding the GC results alone to the clinical-radiologic diagnosis in MDD2 (17\% to $29 \%$ ) despite having high overall agreement between the GC and the final MDD2 diagnosis of UIP or non-UIP (92\%). We think that MDD2 was slightly less swayed by the GC data than MMD1 and opine that this may be related to the nextgeneration sequencing research experience of members of MDD1.

BLC and GC had different impacts on the MDD consensus diagnosis based on classification of the HRCT imaging as probable vs indeterminate for UIP. When GC was introduced following BLC in MDD1, there was no increase in the proportion of high-confidence
Figure 3 - Cohen's $K$ and overall agreement of categorical clinical diagnosis of IPF or non-IPF between the genomic classifier and histopathology in each group with the final multidisciplinary discussion diagnosis in the same group. GC= genomic classifier. See Figures 1 and 2 legends for expansion of other abbreviations.

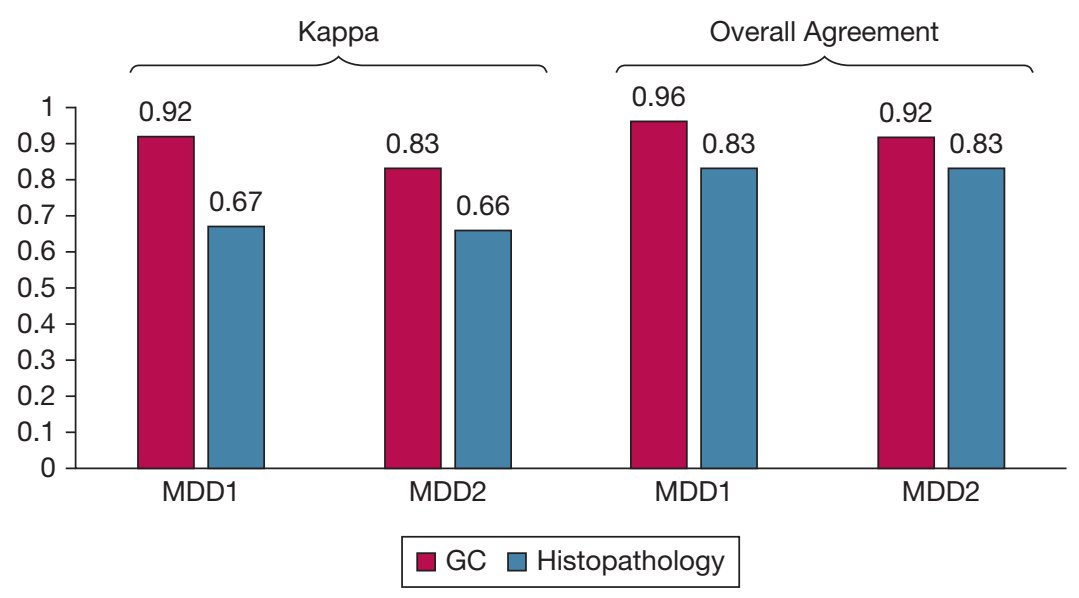




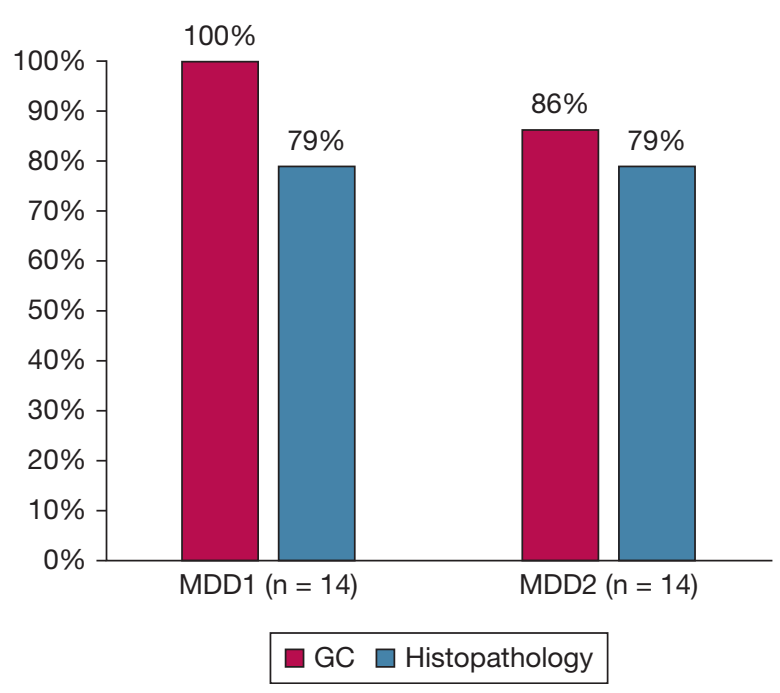

Figure 4 - Overall agreement of categorical clinical diagnosis of IPF or non-IPF between the genomic classifier and histopathology in patients with a radiologic pattern of UIP or probable UIP with the final MDD diagnosis in the same group. See Figure 1 and 2 legends for expansion of abbreviations.

diagnoses among patients with a radiologic pattern most consistent with a non-UIP diagnostic pattern. However, when BLC was introduced following GC, there was a considerable increase in the proportion of highconfidence diagnoses for patients with an atypical radiologic pattern for UIP. Thus, GC appeared to be less helpful when the HRCT pattern was inconsistent with UIP, which may be explained in part by the proportion of diagnoses that were not IPF in this group.

Furthermore, the specificity of GC is $88 \%,{ }^{5}$ and that is partially because of false positive results in some patients with CHP. In our study, we had one patient with a positive GC and a final diagnosis of CHP, and thus GC should be used as a component of the MDD work along with other clinical data, in recognition that, as with surgical biopsy specimens, a UIP pattern is sometimes observed with CHP.

This study also demonstrated that GC increased diagnostic confidence in the multidisciplinary diagnosis of ILD. It also showed that the proportion of highconfidence diagnoses increased significantly when GC was provided after BLC as well as when BLC was provided after GC in MDD2 in patients with a radiologic HRCT pattern that was probable for UIP. However, when HRCT imaging was most consistent with a nonUIP diagnostic pattern, BLC was more impactful for making the final diagnosis in both MDD1 and MDD2. Therefore, this study suggests that GC combined with $\mathrm{BLC}$ can raise the diagnostic confidence in patients with a probable UIP pattern on HRCT imaging, whereas BLC alone may be more useful when HRCT imaging is most consistent with a non-UIP diagnostic pattern with low probability of IPF in the context of an MDD. This is the first study, to our knowledge, showing the application of BLC and GC in a real-world clinical practice setting outside a prospective clinical trial.

The decision to perform an SLB depends on the benefit derived from histopathologic examination balanced against the mortality and morbidity associated with the procedure itself. The average in-hospital mortality for patients undergoing SLB in the United States from 2000 to 2011 was $1.7 \%$ for elective procedures and $16 \%$ for nonelective procedures. ${ }^{12}$ In a recent study, Walsh et al ${ }^{13}$ reported that SLB was requested for only $8.1 \%, 29.6 \%$, and $48.4 \%$ of patients with definite, provisional highconfidence, and provisional low-confidence diagnoses of IPF, respectively, from an international cohort of more than 400 respiratory physicians. Thus, SLB is used in only a minority of patients with suspected IPF in clinical practice. Also, SLB can have discordant results from either inadequate sampling or nondiagnostic histopathology even when it is compared with explanted lung following lung transplantation in up to $12 \%$ of cases. ${ }^{14}$ On the other hand, BLC within the context of a multidisciplinary discussion and as compared with SLB for ILD is hampered by a technique that has not yet been well standardized. ${ }^{15,16}$ However, as reported recently in the COLDICE trial, histopathologic agreement between BLC and SLB was $70.8 \%$, and the diagnostic agreement at MDD was $76.9 \% .{ }^{17}$ Although there has been no direct comparison, the reported mortality for BLC $(0.4 \%)$ is lower than that reported for elective SLB (3.5\%). ${ }^{7,18,19}$ Thus, in our view BLC should be discussed with patients as an alternative to SLB in the diagnostic approach to ILD in selected settings. A patient might undergo BLC as a first diagnostic approach and subsequently undergo SLB if results prove to be nondiagnostic following MDD work, especially in patients with an indeterminate pattern for UIP on HRCT imaging. However, based on its high specificity, adding GC to BLC might decrease the need for SLB in patients with a radiologic pattern of probable UIP and nondiagnostic histopathology, as demonstrated in our study.

This study has some limitations. First, there was no direct comparison of either BLC or GC with SLB and thus the diagnostic accuracy of BLC and GC may not be ascertained in our study. However, the goal of this study was to address confidence and diagnostic agreement of 
an MDD. Such direct comparison was addressed by a recently published trial that compared the diagnostic agreement between SLB and BLC and showed high concordance between the paired biopsy specimens for both histopathologic pattern and the MDD diagnosis. ${ }^{17}$ Second, this study was performed in an academic referral center for ILDs and may not be applicable to other community-based centers. However, this study tried to capture the challenges and complexities of a real-life MDD by having two separate blinded MDDs with experts discussing cases with an overall agreement between final MDD diagnoses of $73 \%(\kappa, 0.77)$, which is similar to the reported agreement among expert MDDs $(\kappa, 0.71) .{ }^{20}$ Third, $16.7 \%$ and $21 \%$ of cases were viewed as unclassifiable following the final work of MDD1 and MDD2, respectively. These rates are comparable to the percentage of unclassifiable cases despite MDD with SLB, which has been reported as ranging from $10 \%$ to $25 \%{ }^{21-24}$ Fourth, the sample size was relatively small; larger studies will be helpful to corroborate our findings. Fifth, the study was not powered to conduct subgroup analysis with appropriate statistical power. Finally, as alluded to in the organizational scheme, J. A. L. has previously published manuscripts involving RNA-Seq, and his confidence in the fidelity of the RNA-Seq data may have introduced bias in MDD1.

\section{Interpretation}

This study demonstrated that GC significantly impacted the diagnostic confidence of physicians practicing in referral centers for ILD. The high level of agreement between a positive GC result for UIP and the final MDD diagnosis for patients with an HRCT scan interpreted as probable for UIP suggests that the GC alone could be used when an HRCT scan is viewed as such. BLC was useful mostly in patients with a non-UIP diagnostic pattern on HRCT imaging. Our study indicates that integrating GC data into the full context of recommended practice allows physicians to increase their confidence in the diagnosis of IPF in patients whose HRCT imaging is viewed as indicating probable UIP.

\section{Acknowledgments}

Author contributions: F. K., A. A., and J. A. $\mathrm{L}$. are the guarantors of the content of the manuscript, including the data and analysis, and participated in data analysis, manuscript writing, and manuscript review; G. J. B., P. D., L. D., R. R., S. S., D. L. S., D. W.: participated in multidisciplinary discussion and manuscript review; M. B., R. A.: participated in data collection and manuscript review.

Financial/nonfinancial disclosures: The authors have reported to CHEST the following: F. K. and J. A. L. have received de minimus compensation from Veracyte for educational presentations. None declared (A. A., G. J. B., P. D., L. D., R. R., S. S., D. L. S., D. W., M. B., R. A.).

\section{References}

1. Hetzel J, Maldonado F, Ravaglia C, et al. Transbronchial cryobiopsies for the diagnosis of diffuse parenchymal lung diseases: expert statement from the cryobiopsy working group on safety and utility and a call for standardization of the procedure. Respiration. 2018;95(3):188200.

2. Tomassetti S, Wells AU, Costabel U, et al. Bronchoscopic lung cryobiopsy increases diagnostic confidence in the multidisciplinary diagnosis of idiopathic pulmonary fibrosis. Am J Respir Crit Care Med. 2016;193(7). 745-742.

3. Ravaglia C, Bonifazi M, Wells AU, et al. Safety and diagnostic yield of transbronchial lung cryobiopsy in diffuse parenchymal lung diseases: a comparative study versus video-assisted thoracoscopic lung biopsy and a systematic review of the literature. Respiration. 2016;91(3):215227.

4. Guenther A, Krauss E, Tello S, et al. The European IPF registry (eurIPFreg): baseline characteristics and survival of patients with idiopathic pulmonary fibrosis. Respir Res. 2018;19(1):141.

5. Raghu G, Flaherty KR, Lederer DJ. Use of a molecular classifier to identify usual interstitial pneumonia in conventional transbronchial lung biopsy samples: a prospective validation study. Lancet Respir Med. 2019;7(6):487-496.

6. Flaherty KR, King TE Jr, Raghu G, et al. Idiopathic interstitial pneumonia: what is the effect of a multidisciplinary approach to diagnosis? Am J Respir Crit Care Med. 2004;170(8):904-910.

7. Raghu G, Remy-Jardin M, Myers JL, et al; American Thoracic Society; European Respiratory Society; Japanese Respiratory Society; Latin American Thoracic Society. Diagnosis of idiopathic pulmonary fibrosis: an official ATS/ERS/JRS/ALAT clinical practice guideline. Am J Respir Crit Care Med. 2018;198(5):e44-e68.

8. Abdelghani R, Thakore S, Kaphle U, Lasky JA, Kheir F. Radial endobronchial ultrasound-guided transbronchial cryobiopsy. J Bronchology Interv Pulmonol. 2019;26(4):245-249.

9. Pankratz DG, Choi Y, Imtiaz U, et al. Usual interstitial pneumonia can be detected in transbronchial biopsies using machine learning. Ann Am Thorac Soc. 2017;14(11):1646-1654.
10. Ryerson CJ, Corte TJ, Lee JS, et al. A standardized diagnostic ontology for fibrotic interstitial lung disease: an international working group perspective. Am J Respir Crit Care Med. 2017;196(10): 1249-1254.

11. Raghu G, Lynch D, Godwin JD, et al. Diagnosis of idiopathic pulmonary fibrosis with high-resolution CT in patients with little or no radiological evidence of honeycombing: secondary analysis of a randomized, controlled trial. Lancet Respir Med. 2014;2(4):277-284.

12. Hutchinson JP, Fogarty AW, McKeever TM, Hubbard RB. In-hospital mortality after surgical lung biopsy for interstitial lung disease in the United States: 2000 to 2011. Am J Respir Crit Care Med. 2016;193(10):1161-1167.

13. Walsh SLF, Lederer DJ, Ryerson CJ, et al. Diagnostic likelihood thresholds that define a working diagnosis of idiopathic pulmonary fibrosis. Am J Respir Crit Care Med. 2019;200(9):1146-1153.

14. Panchabhai TS, Arrossi AV, Highland KB, et al. A single-institution study of concordance of pathological diagnoses for interstitial lung diseases between pretransplantation surgical lung biopsies and lung explants. BMC Pulm Med. 2019;19(1):20.

15. Tomassetti S, Wells AU, Costabel U, et al Bronchoscopic lung cryobiopsy increases diagnostic confidence in the multidisciplinary diagnosis of idiopathic pulmonary fibrosis. Am J Respir Crit Care Med. 2016;193(7):745-752.

16. Romagnoli M, Colby TV, Berthet JP, et al. Poor concordance between sequential 
transbronchial lung cryobiopsy and surgical lung biopsy in the diagnosis of diffuse interstitial lung diseases. Am J Respir Crit Care Med. 2019;199(10):12491256.

17. Troy LK, Grainge C, Corte TJ, et al; Cryobiopsy versus Open Lung Biopsy in the Diagnosis of Interstitial Lung Disease Alliance (COLDICE) Investigators. Diagnostic accuracy of transbronchial lung cryobiopsy for interstitial lung disease diagnosis (COLDICE): a prospective, comparative study. Lancet Respir Med. 2020;8(2) 171-181.

18. Sethi J, Ali MS, Mohananey D, et al. Are transbronchial cryobiopsies ready for prime time? A systematic review and meta-analysis. $J$

Bronchology Interv Pulmonol. 2019;26(1) 22-32.

19. Ravaglia C, Wells AU, Tomassetti S, et al. Diagnostic yield and risk/benefit analysis of trans-bronchial lung cryobiopsy in diffuse parenchymal lung diseases: a large cohort of 699 patients. BMC Pulm Med. 2019;19(1):16.

20. Walsh SL, Wells AU, Desai SR, et al. Multicentre evaluation of multidisciplinary team meeting agreement on diagnosis in diffuse parenchymal lung disease: a case-cohort study. Lancet Respir Med. 2016;4(7):557-565.

21. Ryerson CJ, Urbania TH, Richeldi L, et al. Prevalence and prognosis of unclassifiable interstitial lung disease. Eur Respir J. 2013;42(3):750-757.

22. Ooi A, Iyenger S, Ferguson $J$, et al. VATS lung biopsy in suspected, diffuse interstitial lung disease provides diagnosis, and alters management strategies. Heart Lung Circ. 2005;14(2): 90-92.

23. Troy L, Glaspole I, Goh N, et al. Prevalence and prognosis of unclassifiable interstitial lung disease. Eur Respir J. 2014;43(5):1529-1530.

24. Hyldgaard C, Bendstrup E, Wells AU, et al. Unclassifiable interstitial lung diseases: clinical characteristics and survival. Respirology. 2017;22(3):494500. 